\title{
Sodium Phenylacetate
}

National Cancer Institute

\section{Source}

National Cancer Institute. Sodium Phenylacetate. NCI Thesaurus. Code C66723.

The sodium salt form of phenylacetate with ammonia detoxifying activity. Upon administration, sodium phenylacetate binds to glutamine thereby forming phenylacetylg lutamine and is thus excreted by the kidneys. By serving as an alternative to urea for the excretion of waste nitrogen, sodium phenylacetate is able to lower ammonia levels in the blood and prevent hyperammonemia. 